\title{
Randomized prospective trials to study effects of reduced antibiotic usage in abdominal surgery in cows
}

\author{
R. Jorritsma, ${ }^{* 1}$ I. M. van Geijlswijk, † and M. Nielen* \\ ${ }^{*}$ Farm Animal Health Department, Faculty of Veterinary Medicine, Utrecht University, Yalelaan 7, $3584 \mathrm{CL}$, Utrecht, the Netherlands \\ †Pharmacy Department, Faculty of Veterinary Medicine, Utrecht University, Yalelaan 106, 3584 CM, Utrecht, the Netherlands
}

\begin{abstract}
Societal concerns about antibiotic resistance prompted us to evaluate the use of prophylactic and postoperative antibiotic treatments following cesarean section (CS) and exploratory laparotomy (EL) in a surgery theater within the Farm Animal Health clinic. All procedures were performed by supervised veterinary students for training purposes. Cows undergoing CS $(\mathrm{n}=100)$ received either a prophylaxis with a single 5 -g injection of ampicillin-sodium i.v. only (CSL), or in combination with postoperative i.m. injections of ampicillin-trihydrate $20 \%$ for $3 \mathrm{~d}(10 \mathrm{mg} / \mathrm{kg}$, twice a day; $\mathrm{CSH})$. Cows undergoing EL $(\mathrm{n}=110)$ received either no antibiotic prophylaxis (ELN) or were given a single 5-g injection of ampicillin-sodium i.v. (ELL). The primary outcome measure was healthy recovery after surgery, which we assessed according to the need to treat surgery-related complications within a 10-d follow-up period based on daily clinical observations. Cows in all groups had a normal temperature and feed intake, and a satisfying clinical appearance at the end of the follow-up period. The clinical need to treat cows with antibiotics to deal with postoperative complications was higher for the CS groups than the EL groups. Within both CS and EL groups, the number of complications for each protocol was the same. However, in terms of the secondary outcomes, we observed that CSL cows required, for example, more treatments for mastitis and other diseases unrelated to the surgery than CSH cows (odds ratio 2.8; confidence interval 1.2-7.2). The percentage of infected sutures was higher for ELN cows compared with ELL cows (odds ratio 2.6; confidence interval 1.5-4.9). We estimated that $29 \mathrm{CSH}$ treatments were needed to prevent $1 \mathrm{CS}$ cow with serious surgery-related complications in the CSL group. Likewise, 53 ELL treatments would prevent 1 EL cow with surgery-related complications in the ELN group. We therefore concluded that it is possible to
\end{abstract}

Received November 20, 2017.

Accepted May 23, 2018.

${ }^{1}$ Corresponding author: R.Jorritsma@uu.nl reduce antibiotic prophylaxis in CS and EL cows. The low number cows of clinically detected complications were effectively treated with a postoperative antibiotic intervention at the moment of detection.

Key words: randomized trial, cow, surgery, antibiotics, prophylaxis

\section{INTRODUCTION}

Wound infections and sepsis are the main complications of abdominal surgeries in cows. The judicious use of antiseptic and aseptic procedures, careful tissue handling, and surgical skills to reduce the induction of hematomas and dead space are, in most cases, not sufficient to prevent a certain degree of contamination of the surgery site and probably affect the survival and performance of cows after surgery (Lyons et al., 2013). Additionally, abdominal surgery in cows is usually not performed in a dedicated operating theater; therefore, prophylactic and postoperative antibiotic treatments are frequently used in ambulatory veterinary abdominal surgeries (Chicoine et al., 2008).

The use of antibiotic prophylaxis in human surgery is based on guidelines that are commonly generated by measuring risk factors for postoperative wound infections (Bratzler et al., 2013). Among these risk factors are many aspects related to the suboptimal health of the patient, the duration of the surgery, and the classification of the wound into "clean," "clean-contaminated," "contaminated," and "dirty." In line with this classification of human wounds, exploratory laparotomies (EL) in cows should be classified as clean and cesarean sections (CS) as clean-contaminated. Again, in line with human guidelines, antibiotic prophylaxis in cows is, in general, indicated for clean-contaminated wounds and not for clean wounds. However, the number of veterinary guidelines is very limited compared with the number of human guidelines and compliance appears to be poor (Hardefeldt et al., 2017a).

As a precautionary measure, the prophylactic use of antibiotics is common following abdominal surgery in dairy cattle (Newman and Anderson, 2005; Chicoine et al., 2008). Furthermore, postoperative antibiotic 
treatments are, in our experience, standard procedure though the clinical necessity is not evidence based (Dumas et al., 2016). The growing concerns about the effect of the use of antibiotics on the selection of antibiotic resistance requires an increased focus on the prudent use of antibiotics. The objective of this study was therefore to evaluate the efficacy of 2 antibiotic protocols for EL and CS in dairy cows.

\section{MATERIALS AND METHODS}

\section{Design and Setting}

We performed a randomized parallel study with balanced randomization on cows that were used for the clinical training of veterinary students' surgical skills at Utrecht University's Farm Animal Health clinic. Students following the farm animal track were taught 2 abdominal surgical procedures: EL and CS. From December 2013 to June 2017, all cows submitted to one of these 2 surgical procedures were randomly allocated to one of the antibiotic prophylactic protocols. All procedures, including the use of different antibiotic protocols, were approved by the Ethical Committee of Utrecht University (2013.III.09.062, 2013.III.01.001). We received no external financial support for this research.

\section{Inclusion of Cows}

Some CS cows were inseminated at the clinic, whereas other pregnant cows with a known insemination date were bought for CS and arrived at the clinic throughout the year at 7 or 8 mo pregnant. All these cows underwent standard monitoring, with their blood progesterone concentrations measured 3 times a week and clinical observations by the clinic's veterinary students and staff. To ensure that all CS procedures could be taught during working hours, we frequently induced parturition using hormonal induction. This hormonal induction was based on prodromal signs and was initiated only when the calculated pregnancy had lasted for $265 \mathrm{~d}$ or longer. Induction consisted of a single injection of dexamethasone $(0.06 \mathrm{mg} / \mathrm{kg}$ of $\mathrm{BW})$ approximately $36 \mathrm{~h}$ before the scheduled CS. At the discretion of the clinician, this was sometimes followed by an injection of prostaglandins $(25 \mathrm{mg}$ ) approximately $12 \mathrm{~h}$ before the CS. Some cows were in partu earlier than expected and were submitted to CS before the hormonal induction had been completely finished or even started. When deemed necessary, i.v. injections of clenbuterol hydrochloride $(0.3 \mathrm{mg} / \mathrm{cow})$ were applied for uterus relaxation. Although vaginal delivery of the calf was precluded, the presence of vaginal dilatation was part of the routine clinical examination before CS and thus recorded. Cows were not included in the study if, for example, they unexpectedly and spontaneously delivered during the night. Cows from the teaching herd were eligible for EL if they were clinically healthy and had not previously undergone right flank laparotomy.

\section{Surgical Preparations and Procedures}

Per protocol, all cows were completely washed by trained technicians, preferably the evening before surgery, but at least $2 \mathrm{~h}$ before entering the preparation room. Students performed a pre-anesthetic clinical examination just before the cows were transferred to the preparation room. The CS and EL cows were prepared for standing flank surgery by clipping and shaving an area on their left flank for CS and on the right flank for EL with the 12th rib and the tuber coxa as horizontal limits. The spinal vertebral processes and a horizontal line at the height of the knee were used as vertical limits.

The surgical site was further prepared using antiseptic washes with $70 \%$ ethanol and chlorhexidine in ethanol $70 \%$ as disinfectants. When a CS cow was in partu, additional epidural anesthesia was given between the first and second sacral vertebrae. Proximal paravertebral anesthesia was used in EL cows and 60 to $80 \mathrm{~mL}$ of local infiltration anesthesia was used in CS cows. Due to changing market availabilities of registered products, different generic products of procaine 2 to $4 \%$ with and without adrenaline were used and dosed according to cascade regulations.

After preparation, the cows were moved to the operating theater. As a rule, 2 inexperienced veterinary students and 1 clinician from among the veterinary staff performed all CS and EL. Before entering the operation room, they put on a cap, surgical mask, and designated clothes and boots. They washed their hands with soap and used $70 \%$ alcohol for disinfection before putting on their surgical gloves and gowns. Cotton covers were used to cover the cows and parts of the cow crush.

In cows undergoing CS, the abdominal cavity was opened on the left flank using straightforward incisions of the skin and all muscle layers, and explored manually. Both students individually tried to externalize the gravid uterine horn, which was finally opened and the calf removed. It was generally not possible to completely prevent intra-abdominal contamination with uterine fluids. The uterine incision was sutured using the Utrecht modified Cushing method (Baird, 1989). The abdominal cavity was closed using absorbable polyglactin (Vicryl, Ethicon, Somerville, NY) for 
continuous suture of the muscular layers and nonabsorbable polyamide (Supramid, B. Braun, Melsungen, Germany) for the Ford interlocking sutures of the skin.

In cows undergoing EL, the abdominal cavity was opened using a straightforward vertical incision of the skin and muscle layers of the right flank, except for a blunt opening of the internal oblique muscle. Two students explored the abdominal cavity, guided by their supervising staff veterinarian. The students were asked to identify the reticulum, the abomasum, the liver, the gall bladder, the kidneys, the uterus, the ovaria, the bladder, the rumen and the spleen, and to exteriorize the cecum and parts of the jejunum. To close the abdominal cavity after repositioning, absorbable polyglactin (Vicryl, Ethicon) was used for the muscular layers, whereas the skin was closed with nonabsorbable nylon (Ethilon, Ethicon) using the Ford interlocking technique.

The total duration and timing of the surgeries was not systematically recorded. Based on anecdotal measurements and experience, the abdomen was usually closed within $2 \mathrm{~h}$, and the total duration of each surgery was approximately $2.5 \mathrm{~h}$.

\section{Randomization and Antibiotic Regimens}

Following arrival in the preparation room, the cows were allocated to 1 of 2 antibiotic regimen groups by a staff technician applying the predefined randomization list compiled by the first author of this paper.

Given the classification of the surgery and the strong recommendation to use antibiotic prophylaxis in human patients submitted for CS, we used antibiotic prophylaxis in both regimens for the CS cows (Smaill and Grivell, 2014). A CS group with low antibiotic use (CSL) received a single 5 -g i.v. injection $(10 \mathrm{mg} / \mathrm{kg}$ of BW) of ampicillin-sodium as surgical prophylaxis only, in accordance with the authorization of the product. This is the only antibiotic drug registered for surgery prophylaxis in dairy cattle in the Netherlands. The CS high (CSH) group was treated in line with the clinic's existing protocol for cows submitted for any abdominal surgery: this group received i.m. injections of ampicillin-trihydrate $20 \%$ twice a day for $3 \mathrm{~d}$ postsurgery $(10 \mathrm{mg} / \mathrm{kg})$ in addition to a single 5 -g i.v. injection of ampicillin-sodium as a surgical prophylaxis.

As we classified the EL as a clean surgery, we decided to use no antibiotic prophylaxis in one of the protocols for this indication (ELN). Cows in the other group (ELL) received a single 5-g i.v. injection of ampicillinsodium as surgical prophylaxis only.

All antibiotic prophylaxes were given just before the surgeons entered the scrub room, which was approximately 15 min before the first incision. After surgery, all groups received a single i.m. injection with meloxicam $(0.5 \mathrm{mg} / \mathrm{kg}$ of $\mathrm{BW})$, which, if necessary, was repeated after $72 \mathrm{~h}$.

\section{Follow-Up and Evaluation}

We defined healthy recovery after CS and EL surgery as our primary outcome. To quantify this, we closely monitored all cows included in the study daily for $10 \mathrm{~d}$ to detect peritonitis or sepsis by observing respiration rate, heart rate, rectal temperature, and general clinical appearance. When deemed necessary, we performed additional clinical examinations and treated cows with suspected peritonitis or sepsis with antibiotics. We used the clinical necessity to use antibiotics within a $10-d$ follow-up period after surgery for either of these complications to quantify the primary outcome.

Antibiotic treatments for other purposes, such as mastitis and endometritis, were not considered as a complication of the surgical procedure. Nevertheless, these antibiotic treatments could contribute to the early treatment of undetected complications due to the surgeries, so we included the combined use of all antibiotics for all indications within the 10-d follow-up period as a secondary outcome.

We also used the percentage of infected sutures per individual cow and a clinical classification of the wound infection as secondary outcomes. To this end, we counted the number of suture punctures with and without clearly visible pus during removal of the skin sutures at approximately 10 to $12 \mathrm{~d}$ after surgery. On the same day, we scored the degree of wound infection into 3 infection categories, independent of possible wound emphysema: no or very mild (score 1), moderate wound infection (score 2), and severe wound infection (score 3).

Students were in charge of all treatments and for the removal and counting of suture punctures with and without visible pus and therefore not blinded to the antibiotic regimen. Likewise, the surgeon supervising the students during the surgery was not blinded. We also aimed to obtain a clinical wound score by a clinician on duty in the clinic who was blinded to the antibiotic regimen. Although this scoring clinician was not responsible for the actual injections with antibiotics and was also not actively informed about the antibiotic protocol when scoring the wound, it is possible that he or she heard rumors and was aware of the treatment protocol. It appeared that in $16 \mathrm{CS}$ cases and $17 \mathrm{EL}$ cases, the surgeon scored the wound of a cow he or she had previously operated on.

We based our sample size calculation on our experience that previous protocols resulted in the healthy recovery of at least 90 to $95 \%$ of all CS and EL cows. 
Given an allocation ratio of $50 \%$, around 90 to 100 observations per surgery type were required to find that the percentage of cows with a healthy recovery was lower than $65 \%$, using $\alpha$ of 0.05 and a power $(1-\beta)$ of 0.80 .

\section{Statistical Analyses}

We used logistic regression models to evaluate the differences between the protocols within both CS and EL groups. Antibiotic use for treatment of surgical complications, total antibiotic use for any indication, and wound score were used as binary dependent variables. The percentage of infected suture punctures were used as grouped binomial variables in the same models, where group was the total number of sutures per cow. Treatment protocols were forced into the models as fixed effects and the surgeon and scoring clinician were also evaluated as fixed and random effects. Furthermore, whether or not the clinical scoring was performed by the surgeon was evaluated as an independent variable. For CS cows, the presence of vaginal dilatation was also tested as an independent variable. After reducing the model according to Akaike's information criterion, we calculated odds ratios from the obtained model estimators. For binary dependent variables, the number needed to treat (NNT) was calculated as 1/ (risk difference) from the number of observed cases per protocol. This parameter therefore represents the absolute number of animals that need to be treated in a high antibiotic dosage group (ELL and CSH) to prevent one additional unwanted outcome (complication within $10 \mathrm{~d}$, wound infections, infected sutures) compared with the lower antibiotic dosage group (ELN and CSL). A low NNT means a prevention is very effective, a high NNT means that many animals need to receive ELL or CSH to prevent a single additional animal with complications.

\section{RESULTS}

A total of 100 CS and 110 EL cows were included in the study and all were declared healthy at the end of the observation period of $10 \mathrm{~d}$. Due to the incompleteness of some observations, the number of observations is not equal for all scores within a treatment protocol. For 2 ELL and 3 ELN cows, although the number of infected sutures was documented, the total number of sutures was not. To enable these observations to remain in the data set, the total number of removed sutures for these cows was set at the average of 12 . No significant effects of surgeon ( $\mathrm{n}=8$ for CS; $\mathrm{n}=10$ for EL) or scoring clinician were found in any of the logistical models.

\section{Cesarean Section}

The results for the CS cows are shown in Table 1. We found no statistical differences between CSL and $\mathrm{CSH}$ in terms of complications and wound score. The presence of vaginal dilatation was not significant in any of the models. More overall postoperative antibiotic treatments were needed in CSL cows (odds ratio = 2.8; CI 1.2-7.2) compared with CSH cows. Although the treatment protocol had no significant effect on the percentage of infected sutures per cow, the scoring clinicians found lower percentages of infected sutures when scoring their own work (odds ratio $=0.34$; CI 0.14-0.7). We calculated that the number of cows that needed to be treated to bring about the different outcomes varied between 4.6 and 29 , depending on the outcome variable (Table 1).

Table 1. Descriptive results of cows submitted for cesarean section with different antibiotic prophylaxis (CSL and $\mathrm{CSH}$ )

\begin{tabular}{|c|c|c|c|}
\hline Variable & $\mathrm{CSL}^{1}$ & $\mathrm{CSH}^{2}$ & $\mathrm{NNT}^{3}$ \\
\hline Complication, ${ }^{4} \%$ (no.) & $\begin{array}{l}21.7 \\
(46)\end{array}$ & $\begin{array}{l}18.4 \\
(49)\end{array}$ & 29 \\
\hline Needed antibiotic treatment, ${ }^{5} \%$ (no.) & $\begin{array}{l}42.5 \\
(47)\end{array}$ & $\begin{array}{l}20.8 \\
(48)\end{array}$ & 4.6 \\
\hline Wound score $\geq 2, \%$ (no.) & $\begin{array}{l}32.4 \\
(37)\end{array}$ & $\begin{array}{l}26.7 \\
(45)\end{array}$ & 17 \\
\hline Median percentage infected sutures, \% (minimum-maximum) & $\begin{array}{l}0 \\
(0-64)\end{array}$ & $(0-100)$ & - \\
\hline \multicolumn{4}{|c|}{${ }^{1}$ Antibiotic prophylaxis with a single 5-g i.v. injection of ampicillin-sodium. } \\
\hline \multirow{2}{*}{\multicolumn{4}{|c|}{$\begin{array}{l}{ }^{2} \text { Antibiotic prophylaxis with a single } 5 \text {-g i.v. injection of } 5 \mathrm{~g} \text { of ampicillin-sodium as surgical prophylaxis an } \\
\text { repeated i.m. injections of ampicillin-trihydrate } 20 \% \text { for } 3 \mathrm{~d}(10 \mathrm{mg} / \mathrm{kg} \text {, twice per day). } \\
{ }^{3} \text { Number needed to treat (NNT) was calculated as } 1 / \text { ( risk difference). }\end{array}$}} \\
\hline & & & \\
\hline \multicolumn{4}{|c|}{${ }^{4}$ The need to treat the cow with antibiotics for peritonitis or sepsis wit } \\
\hline
\end{tabular}


Table 2. Descriptive results of cows submitted for exploratory laparotomy with different antibiotic prophylaxis (ELN and ELL)

\begin{tabular}{lccc}
\hline Variable & $\mathrm{ELN}^{1}$ & $\mathrm{ELL}^{2}$ & $\mathrm{NNT}^{3}$ \\
\hline Complication, ${ }^{4} \%$ (no.) & 1.9 & 0 & 53 \\
Needed antibiotic treatment, ${ }^{5} \%$ (no.) & $(52)$ & $(51)$ & 19 \\
& 1.9 & 7.1 & 19 \\
Wound score $\geq 2, \%$ (no.) & $(54)$ & $(56)$ & 12 \\
Median percentage infected sutures, \% (minimum-maximum) & $(48)$ & $(50)$ & \\
& 0 & 0 & - \\
\hline
\end{tabular}

${ }^{1}$ No antibiotic prophylaxis.

${ }^{2}$ Antibiotic prophylaxis with a single 5-g i.v. injection of ampicillin-sodium.

${ }^{3}$ Number needed to treat (NNT) was calculated as 1/(risk difference).

${ }^{4}$ The need to treat the cow with antibiotics for peritonitis or sepsis within $10 \mathrm{~d}$ after surgery.

${ }^{5}$ All antibiotic use within $10 \mathrm{~d}$ postsurgery for any indication.

\section{Exploratory Laparotomy}

The results for the EL cows are shown in Table 2. We found no statistical differences in terms of the antibiotic treatment protocol on complications, wound score, or the overall postoperative use of antibiotics within 10 d of surgery between ELN and ELL cows. The percentage of infected sutures was higher for ELN compared with ELL (odds ratio $=2.6$; CI 1.5-4.9). The estimated number that needed to be treated to bring about the different outcomes varied between 12 and 53 (Table 2).

\section{DISCUSSION}

This paper describes the effects of 2 antibiotic protocols on 4 clinical parameters related to surgical site infections after CS and EL. In addition to the classification of the surgery, hygiene and many other conditions around the surgery are also known to have an effect on surgical site infections (Dumas et al., 2016). The setting of both surgeries in this study is certainly different from the setting of most farm animal surgeries. First, all EL cows were healthy and did not require any surgical intervention, and all CS were carried out without first attempting vaginal extractions and without prior complications. Second, all the surgeries were performed in an operating theater with a standardized hygienic environment, and not in the open air, or in barns, sheds, or other facilities with varying hygienic conditions. Furthermore, in contrast to many farm practices, the animals in this study were probably on average cleaner, were shaved and disinfected more extensively, and surgeons used a cap, mask, and specially designated boots and clothes when operating. On the other hand, the surgeries in this study were all part of the regular teaching curriculum and characterized by a large number of manipulations by students and took a long time, which probably increased the risk of surgical site infections compared with the situation in clinical practice. As a result, it is impossible to extrapolate the results of this study directly to farm animal clinical practice. However, the results may be used as proof of concept that the restricted use of antibiotics is possible. This study may also be indicative of the differences between the risks of surgical site infections between EL and CS.

Although a survey in regular veterinary practices by Hanzen et al. (2011) on CS reported that in $80 \%$ of the surgeries antibiotic prophylaxis was given intraabdominally, we did not use this method of administrating antibiotics in our study. Instead, the antibiotics were only used according to the manufacturer's instructions. Ampicillin-sodium is the only veterinary medicinal product that is authorized for surgical prophylaxis in cattle, intravenously administered before incision. Although intra-abdominal application during surgery is also advocated in a protocol by Kolkman et al. (2007), no evidence indicates a higher efficacy of intra-abdominal administration of antibiotics (Lyons et al., 2013). Moreover, human studies have demonstrated that although the prophylactic topical administration of antibiotics into the surgical incision during various nonophthalmic procedures is more effective than a placebo, it is not superior to parenteral administration. Furthermore, topical administration does not increase the efficacy of parenteral antibiotics when used in combination as a prophylaxis. On the contrary, local effects might impair cell-mediated immunity, which is associated with a significantly increased rate of wound infection (Moesgaard and Lykkegaard Nielsen, 1988). Therefore, whenever antibiotics are applied as surgical prophylaxis, systemic administration should be preferred above topical administration.

In general, the evidence suggests that postoperative prophylaxis without preoperative prophylaxis was as ineffective as preoperative antibiotics administered 
more than 1 or $2 \mathrm{~h}$ before incision. The infection risk increased as the time interval between the preoperative antibiotic administration and the incision increased, or if the antibiotic was infused after the first incision. Intraoperative re-dosing appeared to reduce the risk of surgical site infections in operations lasting more than $4 \mathrm{~h}$ but only when the preoperative dose was given correctly (Steinberg et al., 2009).

In our study, after a follow-up period of $10 \mathrm{~d}$, all cows that underwent either a CS or EL had a normal temperature and feed intake, and a satisfying clinical appearance and were therefore declared healthy irrespective of their antibiotic treatment protocol. As a healthy recovery is arguably the most important objective of antibiotic prophylaxis, the selection of the appropriate antibiotic protocol may be regarded as secondary. Nevertheless, extrapolating observations in humans to animals, complications in wound healing and surgical site infections should be regarded as painful and undesirable effects of surgery and should be avoided, especially in the educational setting where the patients' need for the surgical intervention is absent. We addressed this issue with the use of meloxicam in all cows.

The effects of the different antibiotic protocols for cows that underwent CS were not statistically significant in all the 4 selected evaluation criteria. We found that CSL cows were more prone than CSH cows to receive additional antibiotic therapy irrespective of the indication, whereas health problems attributed to the surgery, effects on wound score, and the percentage of infected sutures were not significantly different between the 2 protocols. The inconsistency of the results may be attributed to the additional effect of the postoperative antibiotic treatments on the presence of common periparturient infections in cows, such as mastitis and metritis. The $3 \mathrm{~d}$ of additional antibiotic treatment given to the CSH cows may have prevented those infections from becoming clinically evident and thus needing separate treatment with antibiotics. We estimated that 29 cows needed to be treated according to the CSH protocol to prevent one cow from developing surgeryrelated complications. The question is whether this effect justifies an estimated 28 extra cows being unnecessarily treated with ampicillin-trihydrate for $3 \mathrm{~d}$, whereas a dedicated secondary antibiotic intervention in the group without initial postoperative treatment was apparently equally effective for the final recovery of the cow with surgical complications. We acknowledge that a careful follow-up of patients is essential for the early detection and subsequent adequate treatment of complications. If this follow-up is not be feasible, it may be necessary to accept the unnecessary treatment of some patients.
Although we tried to ensure that the clinician responsible for scoring the wound was blinded to the treatment protocol, this proved to be logistically challenging and was not always possible. In an attempt to address this issue, we included the possible absence of blinding in our analyses. For CS cows, we found that clinicians gave favorable scores to wounds on animals they operated on, which underlines the necessity of blinding.

As vaginal dilatation may result in uterine contamination after the incision of an incompletely exteriorized uterus, we hypothesized that this could contribute to an increase in abdominal contamination and inflammation. However, this effect appeared not to be significant, which may be due to the short time lapse between cervical dilatation and surgery.

Given the results of the bacteriological culture of uterine fluids in cows during CS, the procedure should be classified as clean-contaminated (Mijten et al., 1997). Although we are unaware of clinical trials on the prophylactic use of antibiotics in CS in cows, others have studied the effects of antibiotic use in cows submitted for surgeries classified as clean-contaminated or contaminated. The extension of the perioperative use of antibiotics from 1 to $4 \mathrm{~d}$ postpartum reduced the number of wound infections in calves operated on for a complicated hernia (for example, Klein and Firth, 1988b). In addition to the differences between the surgeries, this may also illustrate the importance of risk factors other than the wound classification, such as the general physical condition and nutritional state of the patient. Indeed, in contrast to the CS cows in this study, the calves in the Klein and Firth (1988b) study showed signs of inflammation even before surgery. In a study evaluating antibiotic prophylaxis for rumenotomy in cattle, a single antibiotic injection with penicillin $G$ immediately before surgery appeared to be equally effective as a protocol with a 7-d postoperative antibiotic treatment (Haven et al., 1992). As in our study, Haven et al. (1992) found that the risk of abscesses, pyrexia, and decreased daily feed intake were not significantly different between the 2 treatment groups, but the risks were numerically lower in the group received antibiotic prophylaxis.

Before the start of this trial, we treated most EL cows with $5 \mathrm{~g}$ of ampicillin-sodium as a surgical prophylaxis and repeated i.m. injections of ampicillin-trihydrate $20 \%$ for 3 d. Similar strategies were used in an Australian study, which reported the prolonged use of antibiotic prophylaxis in nearly all surgical corrections of abomasal displacement (Hardefeldt et al., 2017b). As these surgeries should be classified as clean, in general, antibiotic prophylaxis is not needed. Given the many manipulations and the long duration of the surgeries, we nevertheless decided to use a protocol with a single 
antibiotic prophylaxis and compared this to a protocol without antibiotic use. We found that the effects of the treatment protocol were minimal, as only the percentage of infected sutures was significantly higher in ELN compared with ELL cows (odds ratio $=2.6$ ). In general, the estimated number of cows that we needed to treat to prevent one additional extra complication was higher for EL treatment groups than CS treatment groups. We estimated that antibiotic prophylaxis of 53 EL cows is needed to prevent serious surgery-related complications in one cow. We suggest that this preventive treatment is unnecessary, as the individual treatment of the one infected cow after surgery is equally effective. This is in line with the results of Klein and Firth (1988a), who evaluated the risk of surgical site infections in calves with an uncomplicated umbilical hernia and concluded that it is possible to withdraw antibiotic prophylaxis without increasing the number of surgical site infections.

\section{CONCLUSIONS}

Although this trial was carried out in a special clinic and the surgeries were performed by veterinary students, it shows that a reduction in preventive antibiotic treatments in CS and EL cows is possible without increasing the number of complications. We estimated that we would need 29 antibiotic treatments postsurgery to prevent $1 \mathrm{CS}$ cow with complications in the CSL group and 53 prophylactic antibiotic treatments to prevent $1 \mathrm{EL}$ cow with complications in the ELN group, while dedicated secondary antibiotic interventions appeared equally effective for the final recovery of the cows with complications after surgery.

\section{ACKNOWLEDGMENTS}

The authors thank H. Aardema, G. A. Hooijer, P. Hut, A. P. Koets, J. M. Parlevliet, D. Scholten, A. Vanhoudt, P. L. A. M. Vos, and all students with the Farm Animal Department, Utrecht University, for their excellent contribution to this clinical trial. J. van de Broek (Farm Animal Department, Utrecht University) is acknowledged for his contribution to the statistical analysis. We received no external funding for this project. The authors declare no conflicts of interest. RJ coordinated the data collection and organized the statistical analyses. MN and RJ designed the study and IvG selected the treatments. All authors read and approved the final manuscript.

\section{REFERENCES}

Baird, A. N. 1989. Cesarean section in the cow. Pages 235-271 in A. S. Turner and C. W. McIIwraith, ed. Techniques in Large Animal Surgery, 4th ed. Lea \& Febiger, Philadelphia, PA.

Bratzler, D. W., E. P. Dellinger, K. M. Olsen, T. M. Perl, P. G. Auwaerter, M. K. Bolon, D. N. Fish, L. M. Napolitano, R. G. Sawyer, D. Slain, J. P. Steinberg, and R. A. Weinstein. 2013. American Society of Health-System Pharmacists; Infectious Disease Society of America; Surgical Infection Society; Society for Healthcare Epidemiology of America. Clinical practice guidelines for antimicrobial prophylaxis in surgery. Am. J. Health Syst. Pharm. 70:195-283.

Chicoine, A. L., P. M. Dowling, J. O. Boison, and S. Parker. 2008. A survey of antimicrobial use during bovine abdominal surgery by western Canadian veterinarians. Can. Vet. J. 49:1105-1109.

Dumas, S. E., H. M. French, S. N. Lavergne, C. R. Ramirez, L. J. Brown, C. R. Bromfield, E. F. Garrett, D. D. French, and B. M. Aldridge. 2016. Judicious use of prophylactic antimicrobials to reduce abdominal surgical site infections in periparturient cows: Part 1-a risk factor review. Vet. Rec. 178:654-660.

Hanzen, C., L. Theron, and J. Detilleux. 2011. Réalisation de la césarienne dans l'espèce bovine en Europe: l'intervention et ses conséquences. Bull. G.T.V. 62:61-72.

Hardefeldt, L. Y., G. F. Browning, K. Thursky, J. R. Gilkerson, H. Billman-Jacobe, M. A. Stevenson, and K. E. Bailey. 2017a. Antimicrobials used for surgical prophylaxis by companion animal veterinarians in Australia. Vet. Microbiol. 203:301-307.

Hardefeldt, L. Y., G. F. Browning, K. A. Thursky, J. R. Gilkerson, H. Billman-Jacobe, M. A. Stevenson, and K. E. Bailey. 2017b. Crosssectional study of antimicrobials used for surgical prophylaxis by bovine veterinary practitioners in Australia. Vet. Rec. https://doi .org/10.1136/vr.104375.

Haven, M. L., J. J. Wichtel, D. G. Bristol, J. F. Fetrow, and J. W. Spears. 1992. Effects of antibiotic prophylaxis on postoperative complications after rumenotomy in cattle. J. Am. Vet. Med. Assoc. 200:1332-1335.

Klein, W. R., and E. C. Firth. 1988a. Infection rates in clean surgical procedures with and without prophylactic antibiotics. Vet. Rec. 123:542-543

Klein, W. R., and E. C. Firth. 1988b. Infection rates in contaminated surgical procedures: A comparison of prophylactic treatment for one day or four days. Vet. Rec. 123:564-566.

Kolkman, I., S. De Vliegher, G. Hoflack, M. Van Aert, J. Laureyns, D. Lips, A. de Kruif, and G. Opsomer. 2007. Protocol of the Caesarean section as performed in daily bovine practice in Belgium. Reprod. Domest. Anim. 42:583-589.

Lyons, N. A., S. Karvountzis, and T. J. Knight-Jones. 2013. Aspects of bovine caesarean section associated with calf mortality, dam survival and subsequent fertility. Vet. J. 197:342-350.

Mijten, P., A. E. van den Bogaard, M. J. Hazen, and A. de Kruif. 1997. Bacterial contamination of fetal fluids at the time of cesarean section in the cow. Theriogenology 48:513-521.

Moesgaard, F., and M. Lykkegaard Nielsen. 1988. Failure of topically applied antibiotics, added to systemic prophylaxis, to reduce perineal wound infection in abdominoperineal excision of the rectum. Acta Chir. Scand. 154:589-592.

Newman, K. D., and D. E. Anderson. 2005. Cesarean section in cows. Vet. Clin. North Am. Food Anim. Pract. 21:73-100.

Smaill, F. M., and R. M. Grivell. 2014. Antibiotic prophylaxis versus no prophylaxis for preventing infection after cesarean section. Cochrane Database Syst. Rev. CD007482. https://doi.org/10.1002/ 14651858.CD007482.pub3.

Steinberg, J. P., B. I. Braun, W. C. Hellinger, L. Kusek, M. R. Bozikis, A. J. Bush, E. P. Dellinger, J. P. Burke, B. Simmons, and S. B. Kritchevsky. 2009. Trial to reduce antimicrobial prophylaxis errors (TRAPE) study group. Timing of antimicrobial prophylaxis and the risk of surgical site infections: results from the trial to reduce antimicrobial prophylaxis errors. Ann. Surg. 250:10-16. 